\title{
Design of Controllers and Observer-Based Controllers for Time-Delay Singularly Perturbed Systems via Composite Control
}

\author{
Juing-Shian Chiou \\ Department of Electrical Engineering, Southern Taiwan University of Science and Technology, Tainan City, Taiwan \\ Correspondence should be addressed to Juing-Shian Chiou; jschiou@mail.stust.edu.tw
}

Received 15 February 2013; Accepted 11 April 2013

Academic Editor: Xinyu Song

Copyright (C) 2013 Juing-Shian Chiou. This is an open access article distributed under the Creative Commons Attribution License, which permits unrestricted use, distribution, and reproduction in any medium, provided the original work is properly cited.

\begin{abstract}
This paper presents a novel and general approach, which is based on the composite control method, to synthesize the controller and observer-based state feedback to stabilize the singularly perturbed time-delay systems. First, the equivalent models of the original systems and the subsystems reduced via singular perturbation techniques are derived. Through these equivalent models, $O(\varepsilon)$ approximation of the stabilization and observer design for the original systems can be achieved through separate analyses for the slow and fast subsystems via a transformation of block diagonalization.
\end{abstract}

\section{Introduction}

A number of engineering and physical systems are described by high-order difference (differential) equations. It is computationally demanding to simulate the dynamics of such large-scale systems, due to the high dimensionality. While controllers for such models are costly to implement and are sometimes unrealizable, the singular perturbation method provides a powerful tool to overcome such problems. Singular perturbation technique usually deals with two-time-scale systems, where the analysis of the whole system can be split, for "small" values of $\varepsilon$ (or the limit $\varepsilon \rightarrow 0$ ), into analysis of two separate systems presented in the slow and fast time scales $[1,2]$. The main advantage of this method is a significant reduction on the overall computation requirements. Also, considering the fact that time delay is so frequently encountered in engineering systems [3,4], the stabilization problem of time-delay singularly perturbed systems should be of great interest. Until now, the problem has been only studied in [5-11]. A delay-independent sufficient condition can be given for single time delay in slow states by [5]. A delay-dependent sufficient condition was studied in [6]. Reference [7] proposed a criterion in terms of the $H_{\infty}$ norm to find a delay-dependent stability bound of the multiple time-delay singularly perturbed systems. These results appear to be restricted to continuous time cases and are based on frequency domain techniques. For discrete time cases, [8] utilized the result of [12] to the robustness problem of discrete multiple time-delay singularly perturbed systems for a given $\varepsilon \in\left(0, \varepsilon^{*}\right)$, where $\varepsilon^{*}$ is the exact $\varepsilon$-bound of the discrete singularly perturbed systems. A delay-dependent criterion is proposed to guarantee the robust stability of the system subject to norm-bounded perturbations studied in [9]. The D-stabilization problem of a discrete multiple timedelay singularly perturbed system was investigated in $[10,11]$. Namely, all the stabilization schemes can be realized under the assumption that all states are available for measurements. This assumption does not hold in general; that is, the system's state may be unavailable to implement state feedback. Hence, it is often desirable to design an observer and a controller leading to asymptotic stability of a given equilibrium point.

A key to the stability and stabilization of singularly perturbed systems is the construction of the slow and fast subsystems. It is well known that if the slow and fast subsystems of a linear time-invariant singularly perturbed system are both stable, then the stability of the original (full order) system is guaranteed for sufficiently small $\varepsilon$ [13]. It is somewhat surprising to note that the relationship of controller and observer between the original systems and the reduced systems via singular perturbation techniques has never been justified in the literature. Strictly speaking, $O(\varepsilon)$ approximation of the stabilization for the original systems by 
those of the reduced subsystems in analysis therein requires a mathematical proof in advance. This paper gives such a proof. First, the equivalent models of the singularly perturbed timedelay systems and its reduced subsystems are introduced in this paper. We further develop a transformation of decomposition (block diagonalization) toward the equivalent model based on the so-called reduced technique. Instead of directly proving the relationship of controllers between the original systems and the reduced systems, $O(\varepsilon)$ approximation of the stabilization for the original systems is achieved through separate analyses for the slow and fast subsystems. Secondly, we extend this technique to study the observer and observerbased controller of singularly perturbed time-delay systems. $O(\varepsilon)$ approximation of the observer and observer-based controller for the original systems can also be achieved through separate analyses for the slow and fast subsystems.

The following notations will be used throughout the paper. The identity matrix with dimension $n$ is denoted by $I_{n}$. $\|A\|$ presents the induced 2-norm of matrix $A$; that is, $\|A\|=\operatorname{Max}\left[\lambda\left(A^{T} A\right)\right]^{1 / 2}$.

\section{System Description and Problem Statement}

Consider the following singularly perturbed time-delay systems:

$$
\begin{aligned}
{\left[\begin{array}{c}
\dot{x}_{1}(t) \\
\varepsilon \dot{x}_{2}(t)
\end{array}\right]=} & {\left[\begin{array}{ll}
A_{10} & A_{20} \\
A_{30} & A_{40}
\end{array}\right]\left[\begin{array}{l}
x_{1}(t) \\
x_{2}(t)
\end{array}\right] } \\
& +\left[\begin{array}{ll}
A_{11} & A_{21} \\
A_{31} & A_{41}
\end{array}\right]\left[\begin{array}{l}
x_{1}(t-\tau) \\
x_{2}(t-\tau)
\end{array}\right]+\left[\begin{array}{l}
B_{1} \\
B_{2}
\end{array}\right] u(t) \\
{\left[\begin{array}{c}
x_{1}(0) \\
x_{2}(0)
\end{array}\right]=} & {\left[\begin{array}{l}
x_{1}^{0} \\
x_{2}^{0}
\end{array}\right] } \\
& y(t)=C_{1} x_{1}(t)+C_{2} x_{2}(t)
\end{aligned}
$$

where $x_{1} \in R^{n_{1}}, x_{2} \in R, n=\left(n_{1}+1\right)$ is the order of the whole system, $u \in R^{p}$ is the control, and $y \in R^{m}$ is the output. $A_{i j}, B_{i}, C_{i}$ are constant matrices with appropriate dimensions for $i, j=1,2$. The small positive scale $\varepsilon$ may represent time constants, inertias, masses, or relevant parameters of the corresponding physical systems. $\tau>0$ is the delay time.

Using the methods of the singular perturbation $[1,2]$, that is, setting $\varepsilon=0$, we obtain reduced-order model of systems (1a)-(1b) as follows.

Slow subsystems:

$$
\begin{gathered}
\dot{x}_{1 s}(t)=A_{10} x_{1 s}(t)+A_{20} x_{2 s}(t)+A_{11} x_{1 s}(t-\tau) \\
+A_{21} x_{2 s}(t-\tau)+B_{1} u_{s}(t), \\
0=A_{30} x_{1 s}(t)+A_{40} x_{2 s}(t)+A_{31} x_{1 s}(t-\tau) \\
+A_{41} x_{2 s}(t-\tau)+B_{2} u_{s}(t), \\
y_{s}(t)=C_{1} x_{1 s}(t)+C_{2} x_{2 s}(t),
\end{gathered}
$$

where $x_{1 s}(0)=x_{1}^{0}$ and $x_{2 s}(0)=-A_{40}^{-1} A_{30} x_{1}^{0}-A_{40}^{-1} B_{2} u_{s}(0)$.
Fast subsystems:

$$
\begin{gathered}
\varepsilon \dot{x}_{2 f}(t)=A_{40} x_{2 f}(t)+A_{41} x_{2 f}(t-\tau)+B_{2} u_{f}(t), \\
y_{f}(t)=C_{2} x_{2 f}(t),
\end{gathered}
$$

where $x_{2 f}(0)=x_{2}^{0}-x_{2 s}(0)$.

Remark 1. Note that when the systems (1a)-(1b) are nominal without time delay, that is, $A_{i 1}=0$ for $i=1,2,3,4$, the slow and fast subsystems $(2 \mathrm{a})-(2 \mathrm{e})$ here reduce exactly to those presented in [1].

As we have indicated, no research article ever discussed the relationships of controller between the original systems (1a)-(1b) and the reduced systems (2a)-(2e). For this objective, a helpful lemma is given next.

Consider the linear time-delay system

$$
\dot{x}(t)=A x(t)+B x(t-\tau),
$$

where $x \in R^{n}, A$ and $B$ are matrices in proper dimensions, and $\tau$ is the delay duration.

Lemma 2 (see [14]). The stability of system (3) implies the stability for the following system:

$$
\dot{w}(t)=(A+z B) w(t) \quad \forall|z|=1,
$$

and vice versa.

In the light of Lemma 2, it is obvious that the asymptotic stability of the systems (1a)-(1b) is equivalent to that of the system

$$
\begin{aligned}
{\left[\begin{array}{c}
\dot{w}_{1}(t) \\
\varepsilon \dot{w}_{2}(t)
\end{array}\right]=} & {\left[\begin{array}{cc}
A_{10}+z A_{11} & A_{20}+z A_{21} \\
A_{30}+z A_{31} & A_{40}+z A_{41}
\end{array}\right]\left[\begin{array}{l}
w_{1}(t) \\
w_{2}(t)
\end{array}\right] } \\
& +\left[\begin{array}{l}
B_{1} \\
B_{2}
\end{array}\right] u_{w}(t) .
\end{aligned}
$$

Taking the singular perturbation approach, that is, setting $\varepsilon=0$, yields reduced-order model of system (5) in the following.

Slow subsystems of the system (5):

$$
\begin{gathered}
\dot{w}_{1 s}(t)=A_{w 0}(z) w_{1 s}(t)+B_{w 0}(z) u_{w s}(t), \\
w_{2 s}(t)=-\left(A_{40}+z A_{41}\right)^{-1}\left[\left(A_{30}+z A_{31}\right) w_{1 s}(t)+B_{2} u_{w s}(t)\right],
\end{gathered}
$$

where $A_{w 0}(z)=\left(A_{10}+z A_{11}\right)-\left(A_{20}+z A_{21}\right)\left(A_{40}+\right.$ $\left.z A_{41}\right)^{-1}\left(A_{30}+z A_{31}\right)$ and $B_{w 0}(z)=B_{1}-\left(A_{20}+z A_{21}\right)\left(A_{40}+\right.$ $\left.z A_{41}\right)^{-1} B_{2}$.

Fast subsystems of the system (5):

$$
\varepsilon \dot{w}_{2 f}(t)=\left(A_{40}+z A_{41}\right) w_{2 f}(t)+B_{2} u_{w f}(t) .
$$


Remark 3. The systems (5) and (6a)-(6c) are called the equivalent models of systems (1a)-(1b) and (2a)-(2e), respectively. In view of the previous observation, we only need to examine the relationship of controllers between the system (5) and its reduced systems $(6 a)-(6 c)$.

\section{Main Results}

3.1. The Controller Design. In general, the analysis and design of controller for singularly perturbed bilinear systems can be accomplished by first separating the original systems (1a)-(1b) into the slow subsystem (2a) and fast subsystem (2d) and then obtaining the composite results for systems (1a)-(1b). That is, suppose now that the subsystem controllers, which are to be designed according to the slow and fast mode performance specifications, have the form

$$
\begin{aligned}
& u_{s}(t)=k_{0} x_{1 s}(t), \\
& u_{f}(t)=k_{2} x_{2 f}(t)
\end{aligned}
$$

and the composite control as

$$
u(t)=u_{s}(t)+u_{f}(t) .
$$

Furthermore, the controllers of systems (6a)-(6c) are written as

$$
\begin{aligned}
& u_{w s}(t)=k_{w 0} w_{1 s}(t), \\
& u_{w f}(t)=k_{w 2} w_{2 f}(t)
\end{aligned}
$$

and the composite control of system (5) as

$$
\begin{aligned}
u_{w}(t) & =u_{w s}(t)+u_{w f}(t) \\
& =k_{w 1} w_{1}(t)+k_{w 2} w_{2}(t),
\end{aligned}
$$

where

$$
\begin{aligned}
k_{w 1}= & {\left[I_{p}+k_{w 2}\left(A_{40}+z A_{41}\right)^{-1} B_{2}\right] k_{w 0} } \\
& +k_{w 2}\left(A_{40}+z A_{41}\right)^{-1}\left(A_{30}+z A_{31}\right) .
\end{aligned}
$$

Hence, the closed-loop form of system (5) becomes

$$
\left[\begin{array}{c}
\dot{w}_{1} \\
\varepsilon \dot{w}_{2}
\end{array}\right]=\left[\begin{array}{ll}
A_{w 1} & A_{w 2} \\
A_{w 3} & A_{w 4}
\end{array}\right]\left[\begin{array}{l}
w_{1} \\
w_{2}
\end{array}\right]
$$

where $A_{w 1}=A_{10}+z A_{11}+B_{1} k_{w 1}, A_{w 2}=A_{20}+z A_{21}+$ $B_{1} k_{w 2}, A_{w 3}=A_{30}+z A_{31}+B_{2} k_{w 1}$, and $A_{w 4}=A_{40}+z A_{41}+$ $B_{2} k_{w 2}$.

Theorem 4. Let $k_{0}$ and $k_{2}$ be so designed such that the subsystems (2a)-(2e) are stable. Then, there exists a sufficiently small $\varepsilon_{1}>0$ such that the original systems (1a)-(1b) are stabilizable for any $\varepsilon \in\left(0, \varepsilon_{1}\right]$. Furthermore, such a stabilizing controller can be designed as

$$
\begin{aligned}
u(t)+A_{40}^{-1} A_{41} u(t-\tau)= & A_{u 1} x_{1}(t)+A_{u 2} x_{1}(t-\tau) \\
& +k_{2} x_{2}(t)+k_{2} A_{40}^{-1} A_{41} x_{2}(t-\tau)
\end{aligned}
$$

where $A_{u 1}=k_{0}+k_{2} A_{40}^{-1} B_{2} k_{0}+k_{2} A_{40}^{-1} A_{30}, A_{u 2}=A_{40}^{-1} A_{41}+$ $k_{2} A_{40}^{-1} A_{31}, k_{0}=k_{w 0}$, and $k_{2}=k_{w 2}$.

Proof. The system (12) is already in the standard singularly perturbed form; hence, a complete separation of the system (12) into slow and fast subsystems is achieved by the nonsingular transformation converting the original into blockdiagonal form. Let

$$
\left[\begin{array}{l}
\zeta(t) \\
\eta(t)
\end{array}\right]=\left[\begin{array}{cc}
I_{n_{1}}-\varepsilon H(z) L(z) & -\varepsilon H(z) \\
L(z) & I_{n_{2}}
\end{array}\right]\left[\begin{array}{l}
w_{1}(t) \\
w_{2}(t)
\end{array}\right],
$$

where $L$ and $H$ satisfy

$$
\begin{gathered}
A_{w 4} L-\varepsilon L A_{w 1}+\varepsilon L A_{w 2} L-A_{w 3}=0, \\
\varepsilon\left(A_{w 1}-A_{w 2} L\right) H-H\left(A_{w 4}+\varepsilon L A_{w 2}\right)+A_{w 2}=0 ;
\end{gathered}
$$

we obtain

$$
\left[\begin{array}{c}
\dot{\zeta}(t) \\
\varepsilon \dot{\eta}(t)
\end{array}\right]=\left[\begin{array}{cc}
A_{w 1}-A_{w 2} L & O \\
O & A_{w 4}+\varepsilon L A_{w 2}
\end{array}\right]\left[\begin{array}{l}
\zeta(t) \\
\eta(t)
\end{array}\right] .
$$

For the zero-order approximation solution, let

$$
\begin{aligned}
& L(z)=A_{w 4}^{-1} A_{w 3}+O(\varepsilon), \\
& H(z)=A_{w 2} A_{w 4}^{-1}+O(\varepsilon)
\end{aligned}
$$

systems (17a)-(17b) become

$$
\begin{aligned}
& {\left[\begin{array}{c}
\dot{\zeta}(t) \\
\varepsilon \dot{\eta}(t)
\end{array}\right]} \\
& =\left[\begin{array}{cc}
A_{w 0}+B_{w 0} k_{w 0}+O(\varepsilon) & O \\
O & \left(A_{40}+z A_{41}+B_{2} k_{w 2}\right)+O(\varepsilon)
\end{array}\right] \\
& \quad \times\left[\begin{array}{l}
\zeta(t) \\
\eta(t)
\end{array}\right] .
\end{aligned}
$$

Neglecting the $O(\varepsilon)$ terms, we obtain that the reduced subsystems $(6 a)-(6 c)$ are the $O(\varepsilon)$ approximation of the original system (5).

The block diagram of the systems (1a)-(1b) is shown in Figure 1; that is, the closed-loop form of the original system (8) becomes

$$
\begin{aligned}
{\left[\begin{array}{c}
\dot{x}_{1}(t) \\
\varepsilon \dot{x}_{2}(t) \\
0
\end{array}\right]=} & {\left[\begin{array}{ccc}
A_{10} & A_{20} & B_{1} \\
A_{30} & A_{40} & B_{2} \\
A_{u 1} & k_{2} & -I_{p}
\end{array}\right]\left[\begin{array}{c}
x_{1}(t) \\
x_{2}(t) \\
u(t)
\end{array}\right] } \\
& +\left[\begin{array}{ccc}
A_{11} & A_{21} & 0 \\
A_{31} & A_{41} & 0 \\
A_{u 2} & k_{2} A_{40}^{-1} A_{41} & -A_{40}^{-1} A_{41}
\end{array}\right]\left[\begin{array}{c}
x_{1}(t-\tau) \\
x_{2}(t-\tau) \\
u(t-\tau)
\end{array}\right] .
\end{aligned}
$$




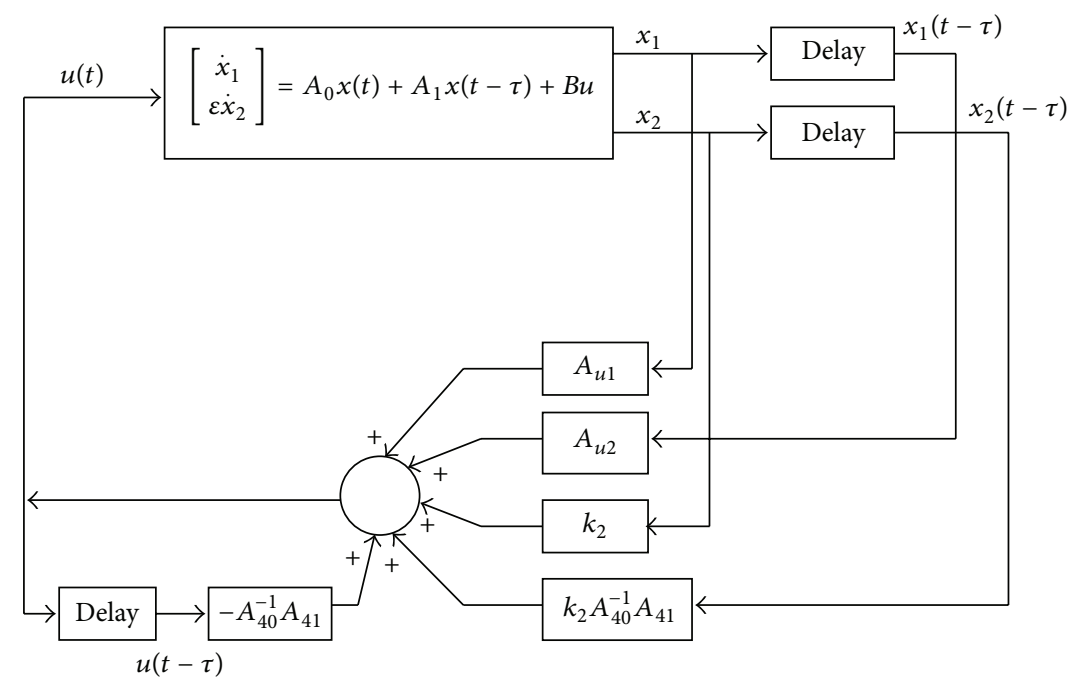

FIGURE 1: Block diagram for the controller design of the systems (1a)-(lb).

Remark 5. Owing to the transformation to diagonal form for systems (1a)-(1b), our approach to show the controller relationship of the singularly perturbed property between the original systems (1a)-(1b) and the reduced systems (2a)-(2e) is somewhat different from those taken in other papers. For systems (5) and (6a)-(6c), the previous theorem shows that, for $\varepsilon_{1}>0$, the stabilization of reduced-order systems (2a)(2e) would guarantee the stabilization of original full-order systems (1a)-(1b). Specifically, the previous proof is based on the following step: the stabilization of the systems (2a)(2e) leads to the stabilization of the systems $(6 a)-(6 c)$, the stabilization of the systems (6a)-(6c) implies the stabilization of the system (5), and the stabilization of the system (5) ensures the stability of the systems (1a)-(1b).

Remark 6. The slow system (2a) is in the standard form of singular system, and the fast system (2c) is a nominal continuous time-delay system. The stabilization of a singular system (2a) and a time-delay system (2c) can be solved by many existing methods [15-18] and [3, 4, 19], respectively. This paper does not focus on the controller design for the reduced subsystems (2a)-(2e), but on the relationship of controller design between the original systems (1a)-(1b) and the reduced systems $(2 a)-(2 e)$.

Remark 7. Note that when the systems (1a)-(1b) are nominal without time delay, that is, $A_{i 1}=0$ for $i=1,2,3,4$, the controller (13) is the same as in [1].

If the systems (1a)-(1b) are autonomous systems, the relationship of stability between the original systems (1a)(1b) and the reduced systems (2a)-(2e) is described in the following corollary.

Corollary 8. If the slow and fast subsystems (2a)-(2e) are both stable, then the original systems (1a)-(1b) are also stable for a sufficiently small $\varepsilon$.
It is sometimes useful to have an upper bound, though conservative, on the value of the small parameter $\varepsilon$ such that Theorem 4 holds. We now give such a bound.

Theorem 9. Under the conditions of Theorem 4, it is valid for all $\varepsilon$ in the interval $0 \leq \varepsilon<\varepsilon_{1}$, where

$$
\varepsilon_{1}=\frac{1}{\bar{\varepsilon}}
$$

which $\bar{\varepsilon}=\max \left[\left\|A_{w 4}^{-1}\right\|\left(\left\|\bar{A}_{w 0}\right\|+\left\|A_{w 2}\right\|\left\|A_{w 4}^{-1} A_{w 3}\right\|+\right.\right.$ $\left.\left.2\left(\left\|\bar{A}_{w 0}\right\|\left\|A_{w 2}\right\|\left\|A_{w 4}^{-1} A_{w 3}\right\|\right)^{1 / 2}\right)\right]$ for all $|z|=1$ and $\bar{A}_{w 0}=$ $A_{w 1}-A_{w 2} A_{w 4}^{-1} A_{w 3}$.

Proof. The proof is similar to that of [1].

Remark 10. Albeit conservative, the previous formula demonstrates the fact that bounds of this sort not only exist but also can be computed. Obtaining a closer bound requires further exploration.

3.2. The Observer-Based Controller Design. The objective of the subsection is to design a full state observer and observerbased feedback for the given systems (1a)-(1b) to be stable. Now, it is required to construct an observer dynamical equation for the original systems (1a)-(1b) as follows:

$$
\begin{aligned}
{\left[\begin{array}{c}
\dot{\hat{x}}_{1}(t) \\
\varepsilon \dot{\hat{x}}_{2}(t)
\end{array}\right]=} & {\left[\begin{array}{ll}
A_{10} & A_{20} \\
A_{30} & A_{40}
\end{array}\right]\left[\begin{array}{l}
\hat{x}_{1}(t) \\
\hat{x}_{2}(t)
\end{array}\right]+\left[\begin{array}{ll}
A_{11} & A_{21} \\
A_{31} & A_{41}
\end{array}\right]\left[\begin{array}{l}
\hat{x}_{1}(t-\tau) \\
\hat{x}_{2}(t-\tau)
\end{array}\right] } \\
& +\left[\begin{array}{l}
B_{1} \\
B_{2}
\end{array}\right] u(t)+\left[\begin{array}{c}
G_{1}(t) \\
G_{2}(\hat{y}(t)-y(t))
\end{array}\right], \\
& \hat{y}(t)=C_{1} \widehat{x}_{1}(t)+C_{2} \widehat{x}_{2}(t) .
\end{aligned}
$$

Furthermore, we obtain the observer of reduced-order model for systems (2a)-(2e) as follows. 
The observer of slow subsystem (2a):

$$
\begin{gathered}
\dot{\hat{x}}_{1 s}(t)=A_{10} \widehat{x}_{1 s}(t)+A_{20} \widehat{x}_{2 s}(t)+A_{11} \widehat{x}_{1 s}(t-\tau) \\
+A_{21} \widehat{x}_{2 s}(t-\tau)+B_{1} u_{s}(t)+G_{0}\left(\hat{y}_{s}-y_{s}\right), \\
0=A_{30} \widehat{x}_{1 s}(t)+A_{40} \widehat{x}_{2 s}(t)+A_{31} \widehat{x}_{1 s}(t-\tau) \\
+A_{41} \widehat{x}_{2 s}(t-\tau)+B_{2} u_{s}(t) \\
\widehat{y}_{s}(t)=C_{1} \widehat{x}_{1 s}(t)+C_{2} \widehat{x}_{2 s}(t) .
\end{gathered}
$$

The observer of fast subsystem (2b):

$$
\begin{aligned}
& \varepsilon \dot{\hat{x}}_{2 f}(t)= A_{40} \widehat{x}_{2 f}(t)+A_{41} \widehat{x}_{2 f}(t-\tau) \\
&+B_{2} u_{f}(t)+G_{2}\left(\hat{y}_{f}-y_{f}\right), \\
& \widehat{y}_{f}(t)=C_{2} \widehat{x}_{2 f}(t) .
\end{aligned}
$$

In the light of Lemma 2, it is obvious that the asymptotic stability of the systems (21a)-(21b) is equivalent to that of the system

$$
\begin{aligned}
& {\left[\begin{array}{c}
\dot{\hat{w}}_{1}(t) \\
\varepsilon \dot{\hat{w}}_{2}(t)
\end{array}\right]=\left[\begin{array}{cc}
A_{10}+z A_{11} & A_{20}+z A_{21} \\
A_{30}+z A_{31} & A_{40}+z A_{41}
\end{array}\right]\left[\begin{array}{c}
\widehat{w}_{1}(t) \\
\widehat{w}_{2}(t)
\end{array}\right]+\left[\begin{array}{l}
B_{1} \\
B_{2}
\end{array}\right] u_{w}(t)} \\
& +\left[\begin{array}{l}
G_{w 1}\left(C_{1} \widehat{w}+C_{2} \widehat{w}-C_{1} w-C_{2} w\right) \\
G_{w 2}\left(C_{1} \widehat{w}+C_{2} \widehat{w}-C_{1} w-C_{2} w\right)
\end{array}\right],
\end{aligned}
$$

and the asymptotic stability of the systems (22a)-(22e) is equivalent to that of the following system.

The observer of slow subsystem (6a):

$$
\begin{aligned}
& \dot{\widehat{w}}_{1 s}(t)= A_{w 0}(z) \widehat{w}_{1 s}+B_{w 0}(z) u_{w s}(t) \\
&+G_{w 0}\left(C_{1} \widehat{w}_{1 s}-C_{2} \widehat{w}_{2 s}-C_{1} w_{1 s}-C_{2} w_{2 s}\right), \\
& \widehat{w}_{2 s}(t)=-\left(A_{40}+z A_{41}\right)^{-1} \\
& \times\left[\left(A_{30}+z A_{31}\right) \widehat{w}_{1 s}(t)+B_{2} u_{w s}(t)\right], \\
& y_{\widehat{w} s}(t)=C_{1} \widehat{w}_{1 s}(t)+C_{2} \widehat{w}_{2 s}(t) .
\end{aligned}
$$

The observer of fast subsystem (6b):

$$
\begin{aligned}
\varepsilon \dot{\widehat{w}}_{2 f}(t)= & \left(A_{40}+z A_{41}\right) \widehat{w}_{2 f}(t)+B_{2} u_{w f}(t) \\
& +G_{w 2}\left(C_{2} \widehat{w}_{2 f}-C_{2} w_{2 f}\right), \\
& y_{\widehat{w} f}(t)=C_{2} \widehat{w}_{2 f}(t) .
\end{aligned}
$$

Using the singular perturbation approach, the observer gain matrix $G_{w 1}$ of the system (23) can be designed as

$$
\begin{aligned}
G_{w 1}= & \left(A_{20}+z A_{21}\right)\left(A_{40}+Z A_{41}\right)^{-1} G_{2} \\
& +G_{0}\left(I_{m}-C_{2}\left(A_{40}+Z A_{41}\right)^{-1} G_{2}\right) .
\end{aligned}
$$

Suppose now that the subsystem controllers, which are to be designed according to the slow and fast mode performance specifications, have the form

$$
\begin{aligned}
& u_{s}(t)=k_{0} \widehat{x}_{1 s}(t), \\
& u_{f}(t)=k_{2} \widehat{x}_{2 f}(t)
\end{aligned}
$$

and the composite control as

$$
u(t)=u_{s}(t)+u_{f}(t) .
$$

Furthermore, the controllers of systems (6a)-(6c) are written as

$$
\begin{aligned}
& u_{w s}(t)=k_{w 0} \widehat{w}_{1 s}(t), \\
& u_{w f}(t)=k_{w 2} \widehat{w}_{2 f}(t)
\end{aligned}
$$

and the composite control of system (5) as

$$
\begin{aligned}
u_{w}(t) & =u_{w s}(t)+u_{w f}(t) \\
& =k_{w 1} \widehat{w}_{1}(t)+k_{w 2} \widehat{w}_{2}(t) .
\end{aligned}
$$

Hence, we obtain the error equations $\left(e_{1}(t)=x_{1}(t)-\right.$ $\left.\widehat{x}_{1}(t), e_{2}(t)=x_{2}(t)-\widehat{x}_{2}(t)\right)$, which can be written as follows:

$$
\begin{aligned}
{\left[\begin{array}{c}
\dot{e}_{1}(t) \\
\varepsilon \dot{e}_{2}(t)
\end{array}\right]=} & {\left[\begin{array}{ll}
A_{10} & A_{20} \\
A_{30} & A_{40}
\end{array}\right]\left[\begin{array}{l}
e_{1}(t) \\
e_{2}(t)
\end{array}\right]+\left[\begin{array}{ll}
A_{11} & A_{21} \\
A_{31} & A_{41}
\end{array}\right]\left[\begin{array}{l}
e_{1}(t-\tau) \\
e_{2}(t-\tau)
\end{array}\right] } \\
& -\left[\begin{array}{c}
G_{1}(t) \\
G_{2}(\hat{y}(t)-y(t))
\end{array}\right]
\end{aligned}
$$

and the error equations $\left(e_{w 1}(t)=w_{1}(t)-\widehat{w}_{1}(t)\right.$ and $e_{w 2}(t)=$ $\left.w_{2}(t)-\widehat{w}_{2}(t)\right)$ of equivalent model of system (5) are as the following:

$$
\left[\begin{array}{c}
\dot{e}_{w 1}(t) \\
\varepsilon \dot{e}_{w 2}(t)
\end{array}\right]=\left[\begin{array}{ll}
A_{w e 1} & A_{w e 2} \\
A_{w e 3} & A_{w e 4}
\end{array}\right]\left[\begin{array}{c}
e_{w 1}(t) \\
e_{w 2}(t)
\end{array}\right]
$$

where $A_{w e 1}=A_{10}+z A_{11}+G_{w 1} C_{1}, A_{w e 2}=A_{20}+z A_{21}+$ $G_{w 1} C_{2}, A_{w e 3}=A_{30}+z A_{31}+G_{w 2} C_{1}$, and $A_{w e 4}=A_{40}+z A_{41}+$ $G_{w 2} C_{2}$.

Furthermore, we obtain the error equations $\left(e_{1 s}(t)=\right.$ $\left.x_{1 s}(t)-\widehat{x}_{1 s}(t), e_{2 f}(t)=x_{2 f}(t)-\widehat{x}_{2 f}(t)\right)$ of the subsystems (2a) $-(2 \mathrm{e})$ that can be written as follows:

$$
\begin{gathered}
\dot{e}_{1 s}(t)=\left(A_{10}+G_{0} C_{1}\right) e_{1 s}(t)+\left(A_{20}+G_{0} C_{2}\right) e_{2 s}(t) \\
+A_{11} e_{1 s}(t-\tau)+A_{21} e_{2 s}(t-\tau), \\
0=A_{30} e_{1 s}(t)+A_{40} e_{2 s}(t)+A_{31} e_{1 s}(t-\tau)+A_{41} e_{2 s}(t-\tau), \\
\varepsilon \dot{e}_{2 f}(t)=\left(A_{40}+G_{2} C_{2}\right) e_{2 f}(t)+A_{41} e_{2 f}(t-\tau),
\end{gathered}
$$

and the error equations $\left(e_{w_{1 s}}(t)=w_{1 s}(t)-\widehat{w}_{1 s}(t)\right.$ and $e_{w_{2 f}}(t)=$ $\left.w_{2 f}(t)-\widehat{w}_{2 f}(t)\right)$ of equivalent model of systems $(6 \mathrm{a})-(6 \mathrm{c})$ are as the following:

$$
\begin{gathered}
\dot{e}_{w_{1 s}}(t)=A_{e w 0} e_{w_{1 s}}(t), \\
\varepsilon \dot{e}_{w_{2 f}}(t)=\left(A_{40}+z A_{41}+G_{w 2} C_{2}\right) e_{w_{2 f}}(t),
\end{gathered}
$$


where $A_{e w 0}=\left(A_{0}+G_{w 0}\left(C_{1}-C_{2}\left(A_{40}+z A_{41}\right)^{-1}\left(A_{30}+\right.\right.\right.$ $\left.\left.\left.z A_{31}\right)\right)\right) e_{w_{1 s}}(t)$.

The corresponding system in the standard singularly perturbed form can be rewritten as sequence

$$
\begin{aligned}
{\left[\begin{array}{c}
\dot{w}_{1}(t) \\
\dot{e}_{w 1}(t) \\
\varepsilon \dot{w}_{2}(t) \\
\varepsilon \dot{e}_{w 2}(t)
\end{array}\right]=} & {\left[\begin{array}{cccc}
A_{w 1} & -B_{1} k_{w 1} & A_{w 2} & -B_{1} k_{w 2} \\
0 & A_{w e 1} & 0 & A_{w e 2} \\
A_{w 3} & -B_{2} k_{w 1} & A_{w 4} & -B_{2} k_{w 2} \\
0 & A_{w e 3} & 0 & A_{w e 4}
\end{array}\right]\left[\begin{array}{c}
w_{1}(t) \\
e_{w 1}(t) \\
w_{2}(t) \\
e_{w 2}(t)
\end{array}\right], } \\
{\left[\begin{array}{c}
\dot{w}_{1 s}(t) \\
\dot{e}_{w 1 s}(t) \\
\varepsilon \dot{w}_{2 f}(t) \\
\varepsilon \dot{e}_{w 2 f}(t)
\end{array}\right]=} & {\left[\begin{array}{cccc}
A_{w 0}+B_{w 0} k_{w 0} & -B_{w 0} k_{w 0} & 0 & 0 \\
0 & A_{w e 0} & 0 & 0 \\
0 & 0 & A_{w 4} & -B_{2} k_{w 2} \\
0 & 0 & 0 & A_{w e 4}
\end{array}\right] } \\
& \times\left[\begin{array}{c}
w_{1 s}(t) \\
e_{w 1 s}(t) \\
w_{2 f}(t) \\
e_{w 2 f}(t)
\end{array}\right] .
\end{aligned}
$$

Theorem 11. Let $G_{0}$ and $G_{2}$ be designed such that the error systems (32a)-(32c) are stable. Then, there exists a sufficiently small $\varepsilon^{*}>0$ such that the original systems (1a)-(1b) are stabilizable for any $\varepsilon \in\left(0, \varepsilon^{*}\right]$. Furthermore, such an observer gain matrix and a stabilizing controller can be designed as

$$
\begin{aligned}
G_{1}(t)+A_{40}^{-1} A_{41} G_{1}(t-\tau)= & A_{G 1}(\widehat{y}(t)-y(t)) \\
& +A_{G 2}(\widehat{y}(t-\tau)-y(t-\tau)), \\
u(t)+A_{40}^{-1} A_{41} u(t-\tau)= & A_{u 1} \widehat{x}_{1}(t)+A_{u 2} \widehat{x}_{1}(t-\tau) \\
& +k_{2} \widehat{x}_{2}(t)+k_{2} A_{40}^{-1} A_{41} \widehat{x}_{2}(t-\tau),
\end{aligned}
$$

where $A_{G 1}=A_{40}^{-1}\left(A_{20} G_{2}+G_{0} A_{40}-G_{0} C_{2} G_{2}\right), A_{G 2}=$ $A_{40}^{-1}\left(A_{21} G_{2}+G_{0} A_{41}\right), G_{0}=G_{w 0}$, and $G_{2}=G_{w 2}$.

Proof. The proof is similar to Theorem 4.

The block diagram of the systems (21a)-(21b) is shown in Figure 2; that is, the closed-loop form of the original systems (1a)-(1b) and the estimation systems (21a)-(21b) becomes

$$
\left[\begin{array}{c}
\dot{x}_{1}(t) \\
\varepsilon \dot{x}_{2}(t) \\
\dot{\vec{x}}_{1}(t) \\
\varepsilon \dot{\bar{x}}_{2}(t) \\
0 \\
0
\end{array}\right]
$$

$$
\begin{aligned}
& =\left[\begin{array}{cccccc}
A_{10} & A_{20} & 0 & 0 & B_{1} & 0 \\
A_{30} & A_{40} & 0 & 0 & B_{2} & 0 \\
0 & 0 & A_{10} & A_{20} & B_{1} & I_{n_{1}} \\
-G_{2} C_{1} & -G_{2} C_{2} & A_{30}+G_{2} C_{1} & A_{40}+G_{2} C_{2} & B_{2} & 0 \\
0 & 0 & A_{u 1} & K_{2} & -I_{P} & 0 \\
-A_{G 1} C_{1} & -A_{G 1} C_{2} & A_{G 1} C_{1} & A_{G 1} C_{2} & 0 & -I_{P}
\end{array}\right] \\
& \times\left[\begin{array}{c}
x_{1}(t) \\
x_{2}(t) \\
\widehat{x}_{1}(t) \\
\widehat{x}_{2}(t) \\
u(t) \\
G_{1}(t)
\end{array}\right]
\end{aligned}
$$$$
+\left[\begin{array}{cccccc}
A_{11} & A_{21} & 0 & 0 & 0 & 0 \\
A_{31} & A_{41} & 0 & 0 & 0 & 0 \\
0 & 0 & A_{11} & A_{21} & 0 & 0 \\
0 & 0 & A_{31} & A_{41} & 0 & 0 \\
0 & 0 & A_{u 2} & K_{2} A_{40}^{-1} A_{41} & -A_{40}^{-1} A_{41} & 0 \\
-A_{G 2} C_{1} & -A_{G 2} C_{2} & A_{G 2} C_{1} & A_{G 2} C_{2} & 0 & -A_{40}^{-1} A_{41}
\end{array}\right]
$$$$
\times\left[\begin{array}{c}
x_{1}(t-\tau) \\
x_{2}(t-\tau) \\
\widehat{x}_{1}(t-\tau) \\
\widehat{x}_{2}(t-\tau) \\
u(t-\tau) \\
G_{1}(t-\tau)
\end{array}\right] .
$$

Remark 12. In view of the transformation to diagonal form for systems (1a)-(1b), our approach to show the observer relationship of the singularly perturbed property between the original system (30) and the reduced systems (32a)(32c) is somewhat different from those taken in other papers. For systems (5) and (6a)-(6c), the previous theorem shows that, for $\varepsilon^{*}>0$, the stabilization of reduced-order systems (32a)-(32c) would guarantee the stabilization of original full-order system (30). Specifically, the previous proof is based on the following step: the stabilization of the systems (32a)-(32c) implies the stabilization of the systems (33a)(33b), the stabilization of the systems (33a)-(33b) implies the stabilization of the system (31), and the stabilization of the system (31) leads to the stability of the system (30).

Remark 13. Note that when the systems (1a)-(1b) are nominal without time delay, that is, $A_{i 1}=0$ for $i=1,2,3,4$, the observer (30) is the same as in [1].

Theorem 14. Under the conditions of Theorem 9, it is valid for all $\varepsilon$ in the interval $0 \leq \varepsilon<\varepsilon^{*}$, where

$$
\varepsilon^{*}=\min \left(\varepsilon_{1}, \varepsilon_{2}\right)
$$




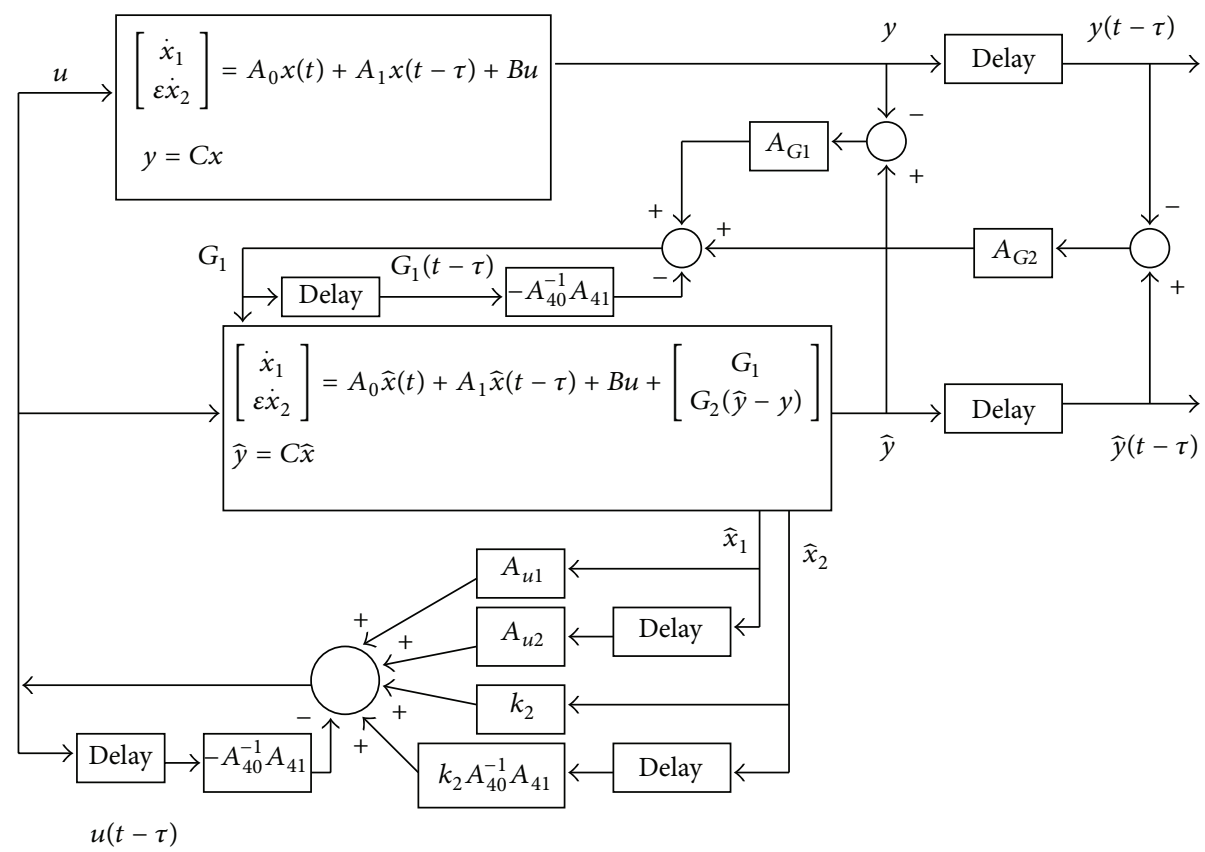

FIGURE 2: Block diagram for the controller and observer design of the systems (1a)-(1b).

which $\varepsilon_{2}=1 / \bar{\varepsilon}_{e}$ and $\bar{\varepsilon}_{e}=\max \left[\left\|A_{w e 4}^{-1}\right\|\left(\left\|A_{w e 0}\right\|+\right.\right.$ $\left.\left.\left\|A_{w e 2}\right\|\left\|A_{w e 4}^{-1} A_{w e 3}\right\|+2\left(\left\|A_{w e 0}\right\|\left\|A_{w e 2}\right\|\left\|A_{w e 4}^{-1} A_{w e 3}\right\|\right)^{1 / 2}\right)\right]$ for all $|z|=1$ and $A_{w e 0}=A_{w e 1}-A_{w e 2} A_{w e 4}^{-1} A_{w e 3}$.

Proof. The proof is similar to that of [1].

\section{Example}

Example 15. Consider the following singularly perturbed time-delay systems:

$$
\begin{aligned}
& {\left[\begin{array}{l}
\dot{x}_{11}(t) \\
\dot{x}_{12}(t) \\
\dot{x}_{13}(t) \\
\varepsilon \dot{x}_{2}(t)
\end{array}\right]} \\
& =\left[\begin{array}{cccc}
0 & -1 & 0 & 1 \\
2 & -1 & 1 & 0 \\
1 & 1 & 0 & 0 \\
0 & 1 & 0 & 1
\end{array}\right]\left[\begin{array}{l}
x_{11}(t) \\
x_{12}(t) \\
x_{13}(t) \\
x_{2}(t)
\end{array}\right]+\left[\begin{array}{cccc}
0.1 & -0.1 & 0 & 0.1 \\
0 & 0.2 & 0 & 0.1 \\
0.1 & 0 & 0 & 0 \\
0 & -0.1 & 0 & -0.25
\end{array}\right] \\
& \times\left[\begin{array}{c}
x_{11}(t-\tau) \\
x_{12}(t-\tau) \\
x_{13}(t-\tau) \\
x_{2}(t-\tau)
\end{array}\right]+\left[\begin{array}{l}
1 \\
1 \\
1 \\
1
\end{array}\right] u(t) \\
& y(t)=\left[\begin{array}{llll}
1 & 1 & 1 & 1
\end{array}\right]\left[\begin{array}{llll}
x_{11}(t) & x_{12}(t) & x_{13}(t) & x_{21}(t)
\end{array}\right]^{T} \text {. }
\end{aligned}
$$

4.1. Controller Design. Consider the following. Slow subsystems:

$$
\begin{aligned}
& {\left[\begin{array}{c}
\dot{x}_{11 s}(t) \\
\dot{x}_{12 s}(t) \\
\dot{x}_{13 s}(t)
\end{array}\right]=\left[\begin{array}{ccc}
0 & -1 & 0 \\
2 & -1 & 1 \\
1 & 1 & 0
\end{array}\right]\left[\begin{array}{l}
x_{11 s}(t) \\
x_{12 s}(t) \\
x_{13 s}(t)
\end{array}\right]+\left[\begin{array}{l}
1 \\
0 \\
0
\end{array}\right] x_{2 s}(t)} \\
& +\left[\begin{array}{ccc}
0.1 & -0.1 & 0 \\
0 & 0.2 & 0 \\
0.1 & 0 & 0
\end{array}\right]\left[\begin{array}{l}
x_{11 s}(t-\tau) \\
x_{12 s}(t-\tau) \\
x_{13 s}(t-\tau)
\end{array}\right] \\
& +\left[\begin{array}{c}
0.1 \\
0.1 \\
0
\end{array}\right] x_{2 s}(t-\tau)+\left[\begin{array}{l}
1 \\
1 \\
1
\end{array}\right] u_{s}(t) \\
& 0=\left[\begin{array}{lll}
0 & 1 & 0
\end{array}\right]\left[\begin{array}{l}
x_{11 s}(t) \\
x_{12 s}(t) \\
x_{13 s}(t)
\end{array}\right]+x_{2 s}(t)+\left[\begin{array}{lll}
0 & -0.1 & 0
\end{array}\right] \\
& \times\left[\begin{array}{c}
x_{11 s}(t-\tau) \\
x_{12 s}(t-\tau) \\
x_{13 s}(t-\tau)
\end{array}\right]-0.25 x_{2 s}(t-\tau)+u_{s}(t), \\
& y_{s}(t)=\left[\begin{array}{lll}
1 & 1 & 1
\end{array}\right] x_{1 s}(t)+x_{2 s}(t) \text {. }
\end{aligned}
$$

Fast subsystems:

$$
\begin{gathered}
\varepsilon \dot{x}_{2 f}(t)=x_{2 f}(t)-0.25 x_{2 f}(t-\tau)+u_{f}(t), \\
y_{f}(t)=x_{2 f}(t) .
\end{gathered}
$$


Suppose now that the subsystem controllers, which are to be designed according to the slow [15] and fast subsystems, have the form

$$
\begin{gathered}
u_{s}(t)=\left[\begin{array}{lll}
-1 & -1 & -1
\end{array}\right] x_{1 s}(t) \\
u_{f}(t)=-4 x_{2 f}(t)
\end{gathered}
$$

where $k_{0}=\left[\begin{array}{lll}-1 & -1 & -1\end{array}\right]$ and $k_{2}=-4$. Therefore, we conclude that system (38) can be stabilized by the following composite control for all $\varepsilon \in(0,1219)$ :

$$
\begin{aligned}
u(t) & -0.25 u(t-\tau) \\
= & {\left[\begin{array}{lll}
3 & -1 & 3
\end{array}\right] x_{1}(t)+\left[\begin{array}{lll}
-0.25 & 0.15 & -0.25
\end{array}\right] x_{1}(t-\tau) } \\
& -4 x_{2}(t)+x_{2}(t-\tau) .
\end{aligned}
$$

4.2. Observer-Based Controller Design. Now, it is required to construct an observer dynamical equation for the original system (38) as follows:

$$
\begin{aligned}
& {\left[\begin{array}{l}
\dot{\hat{x}}_{11}(t) \\
\dot{\hat{x}}_{12}(t) \\
\dot{\hat{x}}_{13}(t) \\
\varepsilon \dot{\hat{x}}_{2}(t)
\end{array}\right]=\left[\begin{array}{cccc}
0 & -1 & 0 & 1 \\
2 & -1 & 1 & 0 \\
1 & 1 & 0 & 0 \\
0 & 1 & 0 & 1
\end{array}\right]\left[\begin{array}{c}
\widehat{x}_{11}(t) \\
\widehat{x}_{12}(t) \\
\hat{x}_{13}(t) \\
\widehat{x}_{2}(t)
\end{array}\right]} \\
& +\left[\begin{array}{cccc}
0.1 & -0.1 & 0 & 0.1 \\
0 & 0.2 & 0 & 0.1 \\
0.1 & 0 & 0 & 0 \\
0 & -0.1 & 0 & -0.25
\end{array}\right]\left[\begin{array}{c}
\widehat{x}_{11}(t-\tau) \\
\widehat{x}_{12}(t-\tau) \\
\widehat{x}_{13}(t-\tau) \\
\widehat{x}_{2}(t-\tau)
\end{array}\right] \\
& +\left[\begin{array}{l}
1 \\
1 \\
1 \\
1
\end{array}\right] u(t)+\left[\begin{array}{c}
G_{1}(t) \\
y(t)-\widehat{y}(t)
\end{array}\right], \\
& \widehat{y}(t)=\left[\begin{array}{llll}
1 & 1 & 1 & 1
\end{array}\right]\left[\begin{array}{llll}
\widehat{x}_{11}(t) & \widehat{x}_{12}(t) & \widehat{x}_{13}(t) & \widehat{x}_{21}(t)
\end{array}\right]^{T} \text {. }
\end{aligned}
$$

Furthermore, we obtain the observer of reduced-order model for system (38) as follows.

The observer of slow subsystem (39a):

$$
\begin{aligned}
{\left[\begin{array}{c}
\dot{\hat{x}}_{11 s}(t) \\
\dot{\hat{x}}_{12 s}(t) \\
\dot{\hat{x}}_{13 s}(t)
\end{array}\right]=} & {\left[\begin{array}{ccc}
0 & -1 & 0 \\
2 & -1 & 1 \\
1 & 1 & 0
\end{array}\right]\left[\begin{array}{l}
\hat{x}_{11 s}(t) \\
\hat{x}_{12 s}(t) \\
\hat{x}_{13 s}(t)
\end{array}\right]+\left[\begin{array}{l}
1 \\
0 \\
0
\end{array}\right] \widehat{x}_{2 s}(t) } \\
& +\left[\begin{array}{ccc}
0.1 & -0.1 & 0 \\
0 & 0.2 & 0 \\
0.1 & 0 & 0
\end{array}\right]\left[\begin{array}{l}
\hat{x}_{11 s}(t-\tau) \\
\hat{x}_{12 s}(t-\tau) \\
\hat{x}_{13 s}(t-\tau)
\end{array}\right] \\
+ & {\left[\begin{array}{c}
0.1 \\
0.1 \\
0
\end{array}\right] \widehat{x}_{2 s}(t-\tau)+\left[\begin{array}{l}
1 \\
1 \\
1
\end{array}\right] u_{s}(t) } \\
+ & {\left[\begin{array}{c}
-4.65 \\
0.24 \\
-0.76
\end{array}\right]\left(\widehat{y}_{s}(t)-y_{s}(t)\right), }
\end{aligned}
$$

$$
\begin{gathered}
0=\left[\begin{array}{lll}
0 & 1 & 0
\end{array}\right]\left[\begin{array}{l}
\widehat{x}_{11 s}(t) \\
\widehat{x}_{12 s}(t) \\
\hat{x}_{13 s}(t)
\end{array}\right]+\widehat{x}_{2 s}(t)+\left[\begin{array}{lll}
0 & -0.1 & 0
\end{array}\right] \\
\times\left[\begin{array}{l}
\widehat{x}_{11 s}(t-\tau) \\
\widehat{x}_{12 s}(t-\tau) \\
\widehat{x}_{13 s}(t-\tau)
\end{array}\right]-0.25 \widehat{x}_{2 s}(t-\tau)+u_{s}(t), \\
\widehat{y}_{s}(t)=\left[\begin{array}{lll}
1 & 1 & 1
\end{array}\right] \widehat{x}_{1 s}(t)+\widehat{x}_{2 s}(t) .
\end{gathered}
$$

The observer of fast subsystem (39d):

$$
\begin{gathered}
\varepsilon \dot{x}_{2 f}(t)=x_{2 f}(t)-0.25 x_{2 f}(t-\tau)+u_{f}(t) \\
+\left(y_{f}(t)-\widehat{y}_{f}(t)\right) \\
\widehat{y}_{f}(t)=\widehat{x}_{2 f}(t)
\end{gathered}
$$

where $G_{0}=\left[\begin{array}{lll}-4.65 & 0.24 & -0.76\end{array}\right]^{T}$ and $G_{2}=-1$.

Therefore, we conclude that system (38) can be stabilized by the following observer-based controller for all $\varepsilon \in(0,0.1)$ :

$$
\begin{aligned}
u(t) & -0.25 u(t-\tau) \\
= & {\left[\begin{array}{lll}
3 & -1 & 3
\end{array}\right] \widehat{x}_{1}(t)+\left[\begin{array}{lll}
-0.25 & 0.15 & -0.25
\end{array}\right] \widehat{x}_{1}(t-\tau) } \\
& -4 \widehat{x}_{2}(t)+\widehat{x}_{2}(t-\tau),
\end{aligned}
$$

where the observer gain can be designed as follows:

$$
\begin{aligned}
G_{1}(t)-0.25 G_{1}(t-\tau)= & {\left[\begin{array}{c}
-10.3 \\
0.48 \\
-1.52
\end{array}\right](\hat{y}(t)-y(t)) } \\
& +\left[\begin{array}{c}
1.0625 \\
-0.16 \\
0.19
\end{array}\right](\hat{y}(t-\tau)-y(t-\tau)) .
\end{aligned}
$$

\section{Conclusion}

For singularly perturbed time-delay systems, this is the first paper that elucidates the relationship of controller and observer design between the original systems and the subsystems obtained by using the singular perturbation reduction techniques. We show that the reduced subsystems are indeed the $O(\varepsilon)$ approximation of the original system. The problems of designing the composite controllers and observers of multidimension fast subsystem and calculating a better bound $\varepsilon$ for singularly perturbed time-delay systems remain open and are interesting topics for future research.

\section{Acknowledgment}

This work is supported by the National Science Council, Taiwan, under Grant nos. NSC101-2221-E-218-027 and NSC1002632-E-218-001-MY3. 


\section{References}

[1] W. Lu, F. Atay, and J. Jost, "Synchronization of discretetime dynamical networks with time-varying couplings," SIAM Journal on Mathematical Analysis, vol. 39, no. 2, pp. 1231-1259, 2007.

[2] D. S. Naidu, Singular Perturbation Methodology in Control Systems, vol. 34 of IEE Control Engineering Series, Peter Peregrinus Ltd., Hitchin, UK, 1988.

[3] H. Wu, "Decentralized adaptive robust control for a class of large-scale systems including delayed state perturbations in the interconnections," IEEE Transactions on Automatic Control, vol. 47, no. 10, pp. 1745-1751, 2002.

[4] Y. Xia and Y. Jia, "Robust sliding-mode control for uncertain time-delay systems: an LMI approach," IEEE Transactions on Automatic Control, vol. 48, no. 6, pp. 1086-1092, 2003.

[5] Z. Shao and J. R. Rowland, "Stability of time-delay singularly perturbed systems," IEE Proceedings: Control Theory and Applications, vol. 142, no. 2, pp. 111-113, 1995.

[6] Z. Shao, "Robust stability of singularly perturbed systems with state delays," IEE Proceedings: Control Theory and Applications, vol. 150, no. 1, pp. 2-6, 2003.

[7] S. T. Pan, F. H. Hsiao, and C. C. Teng, "Stability bound of multiple time delay singularly perturbed systems," Electronics Letters, vol. 32, no. 14, pp. 1327-1328, 1996.

[8] H. Trinh and M. Aldeen, "Robust stability of singularly perturbed discrete-delay systems," IEEE Transactions on Automatic Control, vol. 40, no. 9, pp. 1620-1623, 1995.

[9] C. F. Chen, S. T. Pai, and J. G. Hsieh, "Stability analysis for a class of uncertain discrete singularly perturbed systems with multiple time delay," Journal of Dynamic Systems, Measurement, and Control, vol. 124, no. 9, pp. 467-472, 2002.

[10] F.-H. Hsiao, S.-T. Pan, and C.-C. Teng, "An efficient algorithm for finding the D-stability bound of discrete singularly perturbed systems with multiple time delays," International Journal of Control, vol. 72, no. 1, pp. 1-17, 1999.

[11] F. H. Hsiao, J. D. Hwang, and S. T. Pan, "D-stability problem of discrete singularly perturbed systems," International Journal of Systems Science, vol. 34, no. 3, pp. 227-236, 2003.

[12] T. H. S. Li and J. H. Li, "Stabilization bound of discrete twotime-scale systems," Systems \& Control Letters, vol. 18, no. 6, pp. 479-489, 1992.

[13] Z. H. Shao and M. E. Sawan, "Stabilisation of uncertain singularly perturbed systems," IEE Proceedings: Control Theory and Applications, vol. 153, no. 1, pp. 99-103, 2006.

[14] A. Hmamed, "Further results on the robust stability of uncertain time-delay systems," International Journal of Systems Science, vol. 22, no. 3, pp. 605-614, 1991.

[15] E. Fridman and U. Shaked, "A descriptor system approach to $H_{\infty}$ control of linear time-delay systems," IEEE Transactions on Automatic Control, vol. 47, no. 2, pp. 253-270, 2002.

[16] Z.-H. Guan, G. Chen, X. Yu, and Y. Qin, "Robust decentralized stabilization for a class of large-scale time-delay uncertain impulsive dynamical systems," Automatica, vol. 38, no. 12, pp. 2075-2084, 2002.

[17] E. Fridman and U. Shaked, " $H_{\infty}$-control of linear state-delay descriptor systems: an LMI approach," Linear Algebra and Its Applications, vol. 351-352, pp. 271-302, 2002.

[18] E. Fridman, "New Lyapunov-Krasovskii functionals for stability of linear retarded and neutral type systems," Systems \& Control Letters, vol. 43, no. 4, pp. 309-319, 2001.
[19] C.-H. Chou and C.-C. Cheng, "A decentralized model reference adaptive variable structure controller for large-scale time-varying delay systems," IEEE Transactions on Automatic Control, vol. 48, no. 7, pp. 1213-1217, 2003. 


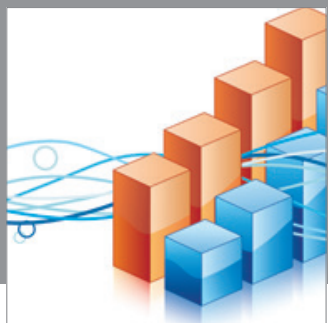

Advances in

Operations Research

mansans

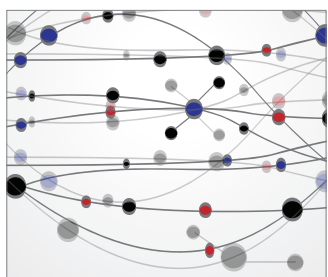

The Scientific World Journal
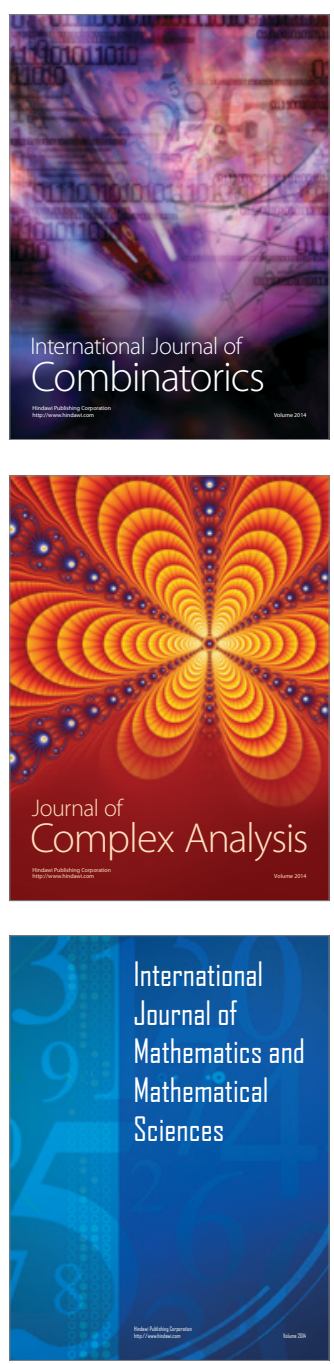
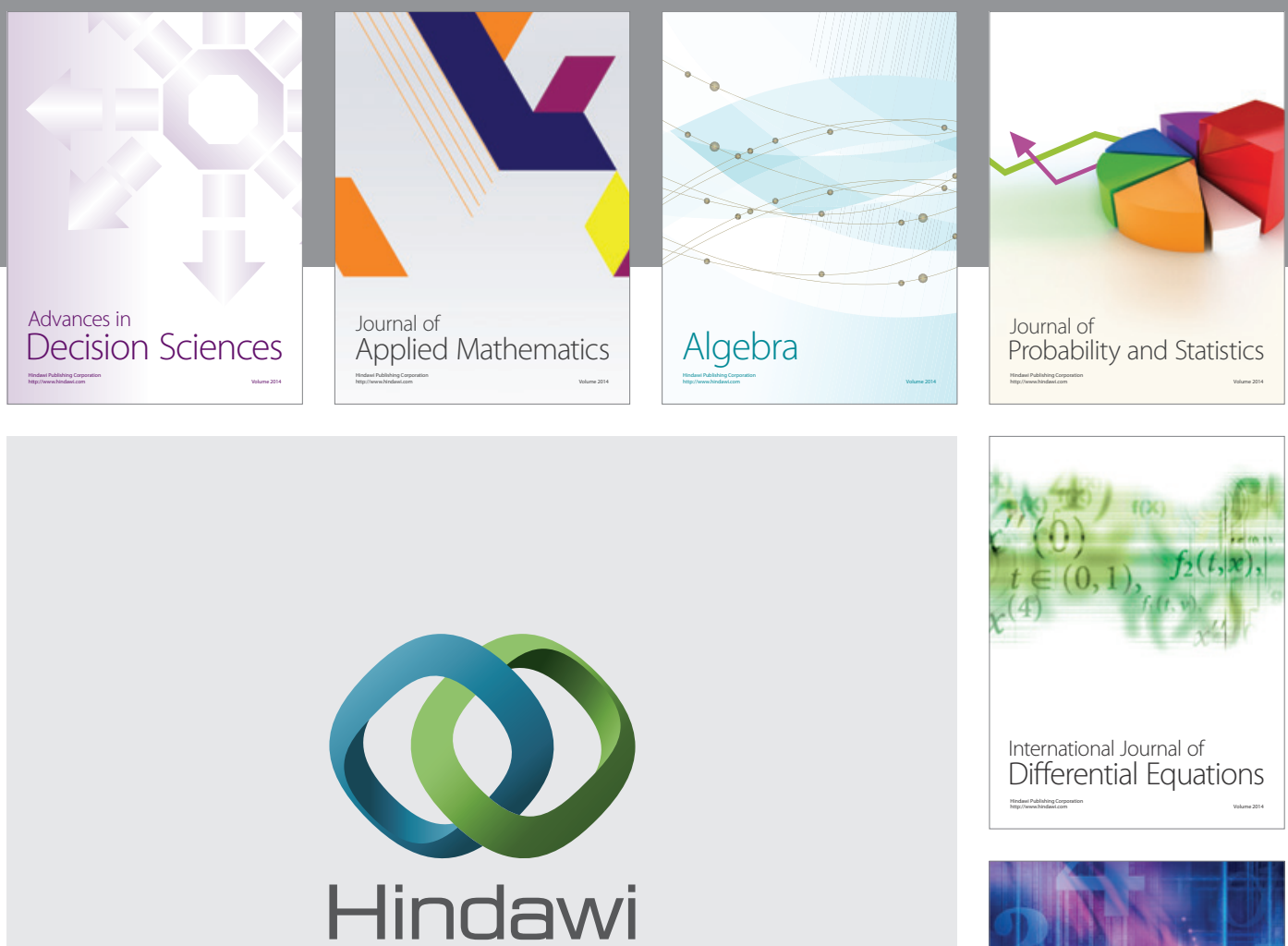

Submit your manuscripts at http://www.hindawi.com
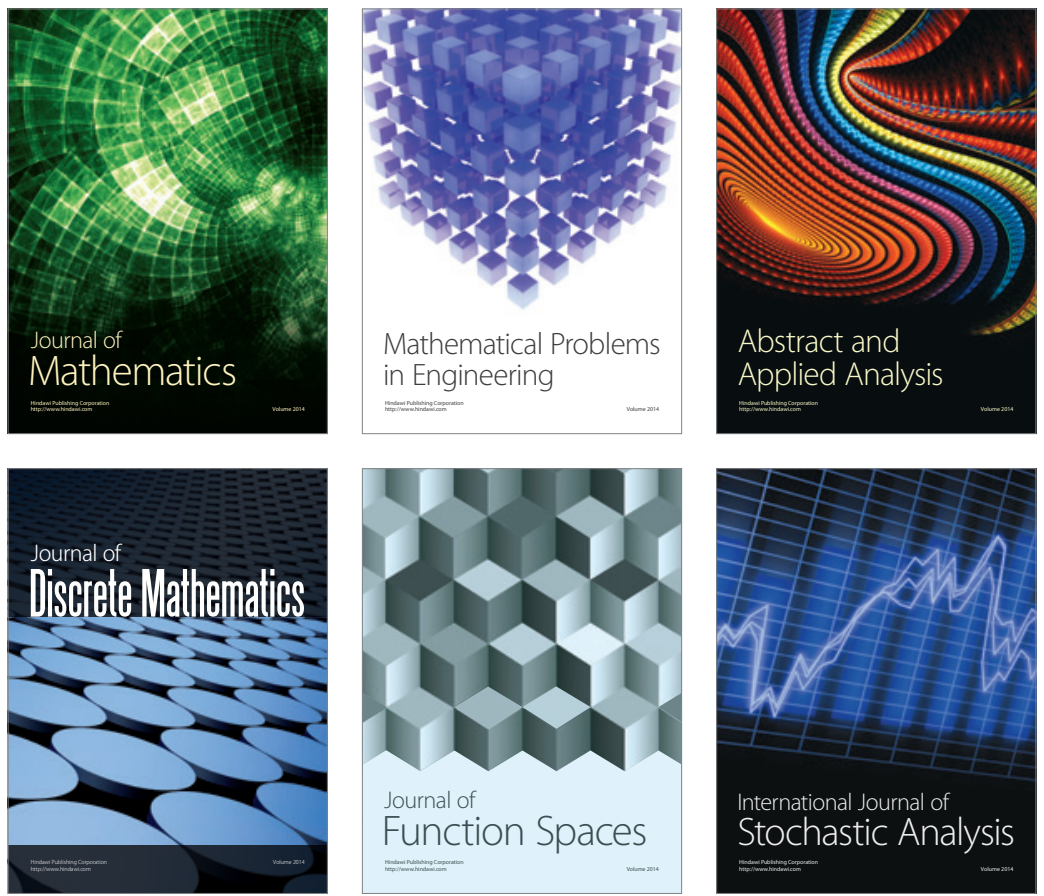

Journal of

Function Spaces

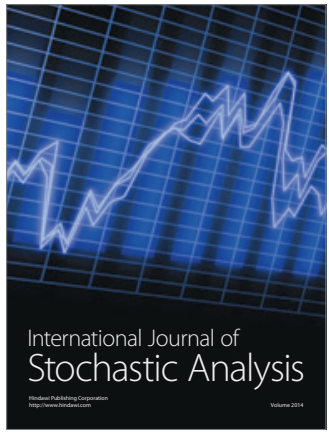

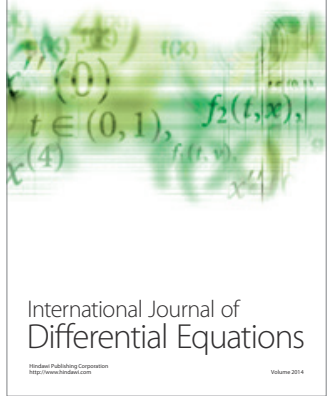
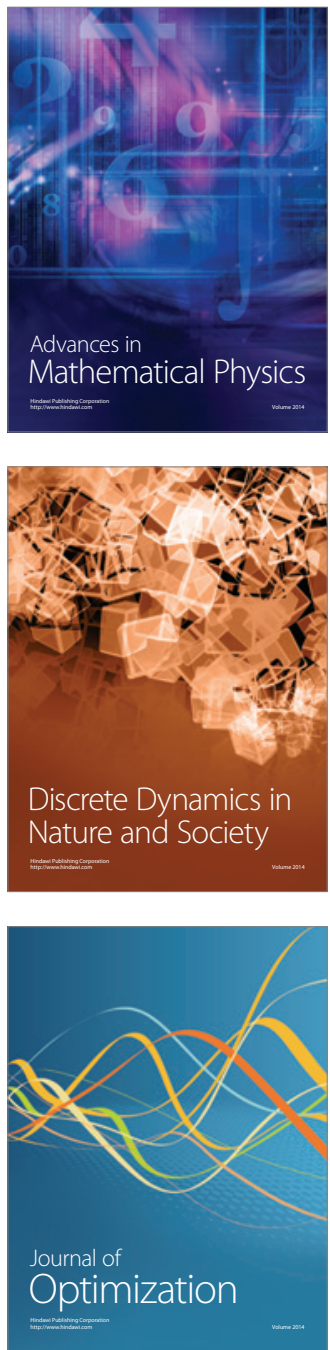